These run times make possible process control using GPC.

The availability of rapid, accurate GPC would be a great boost to polymer research, in studies of both polymerisation and polymer properties. Many effects which are attributed to changes in average molecular weight may turn out to be more directly related to changes in the distribution. In principle, these techniques would also extend the ability of porous gel (Sephadex) columns to separate complete mixtures of proteins; it will be interesting to see if they are taken up.

\section{Aerosol and climate: hotter or cooler?}

\section{by John Gribbin}

THE puzzle of whether an increase in dust in the atmosphere leads to global cooling or global warming is given a further airing in a recent issue of Science (186, 827; 1974). According to some authorities this is a problem of extreme urgency, but the one sure conclusion from the latest results is that it cannot be solved by simple approximations.

Just such a naive estimate led to the original fears that man-made aerosols (that is, small dust particles, not the gases used as propellants in so-called 'aerosol' sprays) might be blocking out enough of the Sun's heat to produce a significant cooling of the Earth, perhaps leading to a new ice age. Volcanic dust had already been suggested to contribute to the onset of natural ice ages, and experiencing a hazy day will suggest to most people that dust in the air reduces the temperature. Although such dust must prevent some of the incident radiation reaching the surface, however, the question remains of how much radiation is absorbed by the dust, and to what extent the dust prevents radiation from the Earth's surface escaping into space. These are non-trivial points, as calculations by Weare, Temkin and Snell emphasise. In simple terms, the problem

ANOTHER recent calculation of the effect of an aerosol layer on the heat balance of the Earth has been reported by Reck (Science, 186, $1034 ; 1974)$. She has looked particularly at the question of whether or not a balance between heating and cooling effects can be achieved, and finds that this is only so for underlying surface albedos in the range 0.35 to 0.60 . For albedos greater than 0.60 , the net effect is always a warming; for surface albedos less than 0.35 the net effect is always a cooling. This effect seems to be independent of the height of the becomes one of whether the 'grey' particles of dust are distributed above a strongly reflecting 'white' ground surface, or above an absorbing 'black' surface. Earlier in 1974 Chýlek and Coakley reported calculations in which an analytical solution of the radiative transfer equation produces a guide to whether aerosol particles will lead to heating or cooling for any given albedo of the underlying surface. The critical ratio is that of the absorption cross section $(1-\omega)$ to the average backscattering cross section $(\omega \beta)$ for the aerosol particles. When this exceeds a certain critical value, heating occurs; when it is less than that value there is cooling. And this critical value is related to the albedo of the underlying system $(a)$ by $(1-\omega) / \omega \beta=(1-a)^{2} / 2 a$, where $\omega$ is the particle single scattering albedo (Science, 183, 75; 1974). This confirms that the same type of aerosol will have different effects over surfaces with different albedos and seemed to provide a guide to how these effects would vary. But the further study by Weare et al. now shows the importance of at least one effect neglected in the earlier calculations.

This is the question of just where in the atmosphere the aerosol layer produces its greatest effect. The relation between aerosol properties and underlying albedo found by Chýlek and Coakley is restricted to the situation when the aerosol layer is distributed above the Earth-atmosphere systemand that is clearly a less than realistic situation. Numerical calculations for the cases when the aerosol layer is above or below the cloud layer, and for a "well mixed" model, show, not surprisingly perhaps, that "at any given preexisting Earth-atmosphere albedo, in order for added aerosol to result in heating, the critical absorptionto-backscatter ratio for the cloud is least when the aerosol is distributed above the cloud". For an annual global average albedo of 0.29 , the critical ratio is 0.9 for an aerosol layer above the cloud, 1.3 in the well mixed model, and 3.2 for an aerosol layer between

aerosol layer above the surface, but too much should not be read into the detailed results. As Reck stresses, the calculations assume average aerosol properties, an average underlying surface albedo, and take no account of the abundances and optical properties of water clouds. Nevertheless, they bear out results of similar but more complex earlier calculations (R. A. Reck, Atmos. Environ., 8, 823; 1974). Reck therefore concludes that this simpler model "is sufficient to determine both the sign and the fact of a finite limit to the heating effect". the cloud and the Earth's surface.

But even these calculations contain sweeping generalisations which require that they be used only with extreme caution in the real world. The "global average" approximation is itself an imperfect guide to reality; the calculations assume diffuse thin cloud rather than a 'lumpy' distribution of cloud cover; and aerosol particles from man's activities do not, by any means, cover the entire globe. The important conclusion from this work is that the effect of added aerosol on the radiation balance of the Earth does indeed depend both on the intrinsic optical properties of the aerosol particles and on their distribution through the atmosphere, as well as on the reflective properties of the underlying surface.

The situation also has an analogy in the problem of how aerosol particles might affect cloud cover. Acting as 'seeds' for droplet formation, they might increase cloud cover, increase the Earth's reflectivity, and produce a global cooling. On the other hand, if the particles simply attach themselves to existing droplets then 'dirty' clouds are produced, which could absorb more radiation and produce a global warming. It seems foolish for mankind to tamper with such a complex and ill understood system, and careless pollution of the atmosphere is unlikely to be a good thing. But we are still far from being able to say with confidence whether or not these polluting activities are hurrying the onset of the coming ice age.

\section{Ocean-continent comparisons}

from Peter J. Smith

The idea has spread rapidly in the Earth sciences that some segments of continent are not truly continental at all, but are of oceanic origin. One of the best known examples of supposedly 'oceanic' continent is that of ophiolite sequences which are thought to be pieces of ancient ocean fioor raised to the surface and sandwiched into continental material during the collision of tectonic plates. The importance of confirming such translocations can hardly be overemphasised, not only because of the plate tectonic implications but hecause it would mean that some oceanic rocks could be studied without the problems of accessibility posed by a thick cover of water. So although the evidence currently available is far from negligible, it is desirable that as many more ways as possible should be found to distinguish between cceanic and continental material. It is thus unfortunate that two potential geochemical differentiators have now proved to be unsuitable. 